\title{
Dose escalation in the era of ablative lung irradiation: is more dose better when it comes to delivery of lung stereotactic body radiation therapy?
}

\author{
Fiori Alite, Anand Mahadevan \\ Department of Radiation Oncology, Geisinger Cancer Institute, Danville, PA, USA \\ Correspondence to: Fiori Alite, MD. Department of Radiation Oncology, Geisinger Cancer Institute, Geisinger Commonwealth School of Medicine, \\ Danville, PA, USA. Email: falite@geisinger.edu. \\ Comment on: Moreno AC, Fellman B, Hobbs BP, et al. Biologically Effective Dose in Stereotactic Body Radiotherapy and Survival for Patients with \\ Early-Stage NSCLC. J Thorac Oncol 2020;15:101-9.
}

Submitted Apr 25, 2020. Accepted for publication Apr 29, 2020.

doi: 10.21037/atm-20-3549

View this article at: http://dx.doi.org/10.21037/atm-20-3549

Dose escalation has been an enduring and elusive target when it comes to improving clinical outcomes for both early stage and locally advanced non-small cell lung cancer (NSCLC). In locally advanced disease a dose threshold of 60 Gy delivered in conventional fractionation together with chemotherapy has been solidified after roughly 50 years of prospective investigation namely due to limitations on normal tissues when treating large thoracic volumes (1). In high risk surgical and medically inoperable stage I NSCLC, the application of four-dimensional image guided planning and delivery of highly conformal stereotactic radiation to small volume parenchymal lung tumors with sharp dose gradients has allowed for safe dose escalation above biologically effective dose (BED) of 100 Gy or achievement of "ablative" radiobiological tumor effects (2). The seminal 2004 publication of a large multiinstitutional analysis of Japanese patients treated with various stereotactic body radiation therapy (SBRT) dose fractionation schedules showed that, when applying the Linear Quadratic formulation to correct for BED with an alpha/beta ratio of 10 , delivering a high $\mathrm{BED}_{10} \geq 100 \mathrm{~Gy}$ significantly improved overall survival (3-year overall survival $88 \%$ vs. 69\%) (3). Experience delivering doses in this range has translated to high rates of local control $(98 \%$ at 2 years) and improved overall survival when compared to conventionally fractionated radiotherapy $(4,5)$. Clinical practice patterns and most institutions have embraced the clear cut-point of $\mathrm{BED}_{10}$ of $100 \mathrm{~Gy}$ to define SBRT lung delivery, but the optimal dose fractionation beyond this point remains unclear and currently employed prescriptions are heterogenous ranging between $100-180$ Gy $\mathrm{BED}_{10}$.

Moreno et al. investigated whether dose escalation beyond $\mathrm{BED}_{10}$ of $100 \mathrm{~Gy}$ imparts improved survival outcomes in patients treated for Stage I NSCLC (6). By retrospectively analyzing the large population based National Cancer Data Base (NCDB), the investigators matched over 20,000 patients treated for Stage I (cT1-T2aN0M0) NSCLC between 2004-2014 and stratified between Low BED ${ }_{10}$ delivery defined as 100-129 Gy and High $\mathrm{BED}_{10}$ delivery defined as $\geq 130$ Gy. The authors were able to demonstrate a modest but statistically significant improvement in overall survival in the patients treated with High BED at both 3 -year (64\% vs. $60 \%)$ and 5 -year (34\% vs. 26\%) ( $\mathrm{P}=0.039)$. On propensity score matched multivariate analysis adjusting for tumor as well as clinical patient characteristics that importantly includes age and comorbidity burden, the improvements in survival remained significant for the high BED arm (HR 0.96, $\mathrm{P}=0.032$ ).

The authors are to be commended for reporting the largest matched cohort to date investigating SBRT dose escalation in early stage NSCLC, employing appropriate statistical rigor to their analysis and controlling for potential available confounders that are coded for in the NCDB. These findings also serve to ballast four other retrospective multi-institutional and population-based database reports demonstrating improved cancer control and survival outcomes with $\mathrm{BED}_{10}$ in the ranges $\geq 125-150 \mathrm{~Gy}$, especially for larger tumor volumes ( $>3 \mathrm{~cm}$ or T2 stage) (7-10). It 
appears that, when feasible and safe, dose escalation beyond $\mathrm{BED}_{10}$ of $130 \mathrm{~Gy}$ seems to be supported by the currently available retrospective experience. It should be noted that consistently lacking in these reports are availability of toxicity data, particularly related to dose.

The significant limitations inherent in comparative efficacy utilizing large dataset analysis, and specifically the NCDB, warrant some scrutiny before we can adapt a strategy that uniformly maximizes $\mathrm{BED}_{10}$. Implicit confounding that cannot be accounted for in the data elements available in the NCDB may in fact influence survival analysis, despite the statistical rigor employed. For example, tumor size and lobe location are coded elements and did not predict survival or influence the High BED improved overall survival effect on multivariate analysis, but tumor location in respect to critical central thoracic, mediastinal or chest wall anatomy is not a coded element and may in fact confound. Tumor centrality as defined by the RTOG (within $2 \mathrm{~cm}$ of the proximal bronchial tree or abutting the mediastinal/ pericardial pleura), ultra-centrality (often defined as tumor abutting mainstem bronchus or carina, trachea, esophagus, great vessels or brachial plexus), or very peripheral tumor locations that overlap the chest wall/ribs influence dose fractionation decisions often leading to lower BED regimens in the interest of sparing normal tissues $(11,12)$. Treating tumors in these critical locations likely drives the shift in SBRT dose regimens patterns of care the authors were able to document over the analysis time period. As the authors clearly demonstrated the significant decline over time of the 60 Gy in 3 fraction regimen and the rise in utilization of $5-10$ fraction regimen coincides with the publications of key reports showing worsening toxicity with 3 fraction courses for central lung tumors (11-13).

By analyzing the dose fractionations utilized in the high BED vs. low BED arms one is able to infer that patients included in the high $\mathrm{BED}_{10}(>130 \mathrm{~Gy})$ arm, likely had fewer central and ultra-central tumor since the majority (71\%) were treated with 3 fraction regimens which are discouraged by multiple consensus guidelines and the NCCN in the timeframe of the analysis (14). When developing dose fractionation regimens and planning radiation delivery in these critical locations, achieving higher equitoxic BEDs comes at the cost of normal tissue toxicity and is often not possible or requires significant tradeoffs of BED, requiring clinical judgment in terms of prioritizing target coverage $v s$. organs at risk (15). In addition to tumor size, central tumor location represents an independent risk factor for occult mediastinal metastases, perhaps portending a more biologically aggressive course after completion of SBRT (higher rates of mediastinal, and/or distant metastatic dissemination) (16). Potential imbalance in the number of patients with central/ultra-central tumors between the high and low $\mathrm{BED}_{10}$ stratified arms may in turn influence the survival analysis.

Even when considering peripheral tumors, the report's premise that higher BED leads to better local control translating into better survival is relatively untested. For example, a systematic review of published prospective SBRT studies does not provide convincing evidence of differences in freedom from local progression in this higher range of EQD2 doses (17). Since the NCDB does not provide cancer specific recurrence endpoints it is also difficult to conclude that the survival gains between the high and low BED arms was clearly secondary to lung cancer specific improvements.

The authors note that their findings are consistent with published randomized trials, but that additional prospective investigation is necessary before we conclude that higher $\mathrm{BED}_{10}$ improves survival. Clearly this is a critical point in light of the multitude of factors that influence patient outcomes when treating early stage NSCLC with SBRT such as delivery technique (conventional linear accelerator based $v s$. robotic platforms), various respiratory motion management strategies (compression, gating or tracking), Image Guided Radiation techniques (fiducial based vs. volumetric imaging vs kilovoltage based imaging), treatment planning considerations around calculation algorithm (pencil beam vs. convolution superposition or Monte Carlo), delivery scheduling (consecutive $v s$. nonconsecutive days), tumor location (central vs. peripheral) as well as emerging literature showing overall poor agreement between results of population based observational studies and subsequent RCTs (18-20).

What will ultimately be required is prospective randomization comparing dose escalated $\mathrm{BED}_{10}$ beyond 130 Gy in peripheral T1-T2 lesions with strict inclusion criteria, radiation delivery quality assurance and normal tissue dose constraints. If clear cancer specific and/or survival gains can be demonstrated with dose escalation in this patient population, prescriptions for treating tumors near higher risk anatomical locations or frailer patients can then be tailored to maximize BED while respecting normal tissue constraints (i.e., $8-15$ hypo-fractionated stereotactic delivery) (21). In addition, knowing if dose escalation above BED of 130 Gy improves OS, would critically inform currently accruing and planned randomized trials comparing SBRT to primary surgical management in 
medically operable patient populations.

Prospective investigation of dose escalation must also be done in synchrony with evolving systemic immunooncology (IO) strategies in patients receiving SBRT for early stage NSCLC, since regional and distant metastatic recurrence is an important competing risk that influences overall survival in this patient population (22). Several clinical trials currently accruing are assessing integration of either adjuvant, post-SBRT PD1 inhibition with durvalumab (PACIFIC 4, NCT03833154) or concurrent and adjuvant integration of PDL1 inhibition with atezolizumab (SWOG S1914, NCT04214262) (23,24). Potential synergy between the immunomodulatory effects of SBRT and check-point inhibition may show this to be a viable strategy and therefore Lower BED fractionation regimens that may be sub-ablative but optimize immune effects and abrogate potential overlapping toxicity profile of IO (i.e., pneumonitis) may need to be explored.

\section{Acknowledgments}

Funding: None.

\section{Footnote}

Provenance and Peer Review: This article was commissioned by the editorial office, Annals of Translational Medicine. The article did not undergo external peer review.

Conflicts of Interest: Both authors have completed the ICMJE uniform disclosure form (available at http://dx.doi. org/10.21037/atm-20-3549). The authors have no conflicts of interest to declare.

Ethical Statement: The authors are accountable for all aspects of the work in ensuring that questions related to the accuracy or integrity of any part of the work are appropriately investigated and resolved.

Open Access Statement: This is an Open Access article distributed in accordance with the Creative Commons Attribution-NonCommercial-NoDerivs 4.0 International License (CC BY-NC-ND 4.0), which permits the noncommercial replication and distribution of the article with the strict proviso that no changes or edits are made and the original work is properly cited (including links to both the formal publication through the relevant DOI and the license). See: https://creativecommons.org/licenses/by-nc-nd/4.0/.

\section{References}

1. Bradley JD, Hu C, Komaki RR, et al. Long-Term Results of NRG Oncology RTOG 0617: Standard- Versus HighDose Chemoradiotherapy With or Without Cetuximab for Unresectable Stage III Non-Small-Cell Lung Cancer. J Clin Oncol 2020;38:706-14.

2. Guckenberger M, Klement RJ, Allgäuer M, et al. Applicability of the linear-quadratic formalism for modeling local tumor control probability in high dose per fraction stereotactic body radiotherapy for early stage nonsmall cell lung cancer. Radiother Oncol 2013;109:13-20.

3. Onishi H, Araki T, Shirato H, et al. Stereotactic hypofractionated high-dose irradiation for stage I nonsmall cell lung carcinoma: clinical outcomes in 245 subjects in a Japanese multiinstitutional study. Cancer 2004;101:1623-31.

4. Timmerman R, Paulus R, Galvin J, et al. Stereotactic body radiation therapy for inoperable early stage lung cancer. JAMA 2010;303:1070-6.

5. Ball D, Mai GT, Vinod S, et al. Stereotactic ablative radiotherapy versus standard radiotherapy in stage 1 nonsmall-cell lung cancer (TROG 09.02 CHISEL): a phase 3, open-label, randomised controlled trial. Lancet Oncol 2019;20:494-503.

6. Moreno AC, Fellman B, Hobbs BP, et al. Biologically Effective Dose in Stereotactic Body Radiotherapy and Survival for Patients with Early-Stage NSCLC. J Thorac Oncol 2020;15:101-9.

7. Kestin L, Grills I, Guckenberger M, et al. Dose-response relationship with clinical outcome for lung stereotactic body radiotherapy (SBRT) delivered via online image guidance. Radiother Oncol 2014;110:499-504.

8. Yan SX, Qureshi MM, Truong MT et al. SBRT for Stage II NSCLC: An Analysis of the National Cancer Database. IJROBP 2017;99:E505-6.

9. Davis JN, Medbery C, Sharma S, et al. Stereotactic body radiotherapy for early-stage non-small cell lung cancer: clinical outcomes from a National Patient Registry. J Radiat Oncol 2015;4:55-63.

10. Koshy M, Malik R, Weichselbaum RR, Sher DJ. Increasing Radiation Therapy Dose Is Associated With Improved Survival in Patients Undergoing Stereotactic Body Radiation Therapy for Stage I Non-Small-Cell Lung Cancer. Int J Radiat Oncol Biol Phys 2015;91:344-50.

11. Timmerman R, McGarry R, Yiannoutsos C, et al. Excessive toxicity when treating central tumors in a phase II study of stereotactic body radiation therapy for 
medically inoperable early-stage lung cancer. J Clin Oncol 2006;24:4833-9.

12. Chang JY, Bezjak A, Mornex F, Committee IART. Stereotactic ablative radiotherapy for centrally located early stage non-small-cell lung cancer: what we have learned. J Thorac Oncol 2015;10:577-85.

13. Corradetti MN, Haas AR, Rengan R. Central-airway necrosis after stereotactic body-radiation therapy. N Engl J Med 2012;366:2327-9.

14. National Comprehensive Cancer Network. Non-small cell lung cancer (version 3.2020). 2020 [accessed 4.19.2020]. Available online: https://www.nccn.org/professionals/ physician_gls/PDF/nscl.pdf

15. Murrell DH, Laba JM, Erickson A, et al. Stereotactic ablative radiotherapy for ultra-central lung tumors: prioritize target coverage or organs at risk? Radiat Oncol 2018;13:57.

16. Lee PC, Port JL, Korst RJ, Available online: Risk factors for occult mediastinal metastases in clinical stage I nonsmall cell lung cancer. Ann Thorac Surg 2007;84:177-81.

17. van Baardwijk A, Tomé WA, van Elmpt W, et al. Is highdose stereotactic body radiotherapy (SBRT) for stage I non-small cell lung cancer (NSCLC) overkill? A systematic review. Radiother Oncol 2012;105:145-9.

18. Latifi K, Oliver J, Baker R, et al. Study of 201 nonsmall cell lung cancer patients given stereotactic ablative radiation therapy shows local control dependence on dose calculation algorithm. Int J Radiat Oncol Biol Phys

Cite this article as: Alite F, Mahadevan A. Dose escalation in the era of ablative lung irradiation: is more dose better when it comes to delivery of lung stereotactic body radiation therapy? Ann Transl Med 2020;8(20):1325. doi: 10.21037/atm-20-3549
2014;88:1108-13.

19. Alite F, Stang K, Balasubramanian N, et al. Local control dependence on consecutive vs. nonconsecutive fractionation in lung stereotactic body radiation therapy. Radiother Oncol 2016;121:9-14.

20. Soni PD, Hartman HE, Dess RT, et al. Comparison of Population-Based Observational Studies With Randomized Trials in Oncology. J Clin Oncol 2019;37:1209-16.

21. Giuliani M, Mathew AS, Bahig H, et al. SUNSET: Stereotactic Radiation for Ultracentral Non-Small-Cell Lung Cancer-A Safety and Efficacy Trial. Clin Lung Cancer 2018;19:e529-32.

22. Miller CJ, Martin B, Stang K, et al. Predictors of Distant Failure After Stereotactic Body Radiation Therapy for Stages I to IIA Non-Small-Cell Lung Cancer. Clin Lung Cancer 2019;20:37-42.

23. Clinicaltrials.gov. National Library of Medicine (US). Durvalumab vs Placebo Following Stereotactic Body Radiation Therapy in Early Stage Non-small Cell Lung Cancer Patients: Identifier: NCT03833154. Available online: https:/clinicaltrials.gov/ct2/show/NCT03833154. [accessed 4.19.2020].

24. Clinicaltrials.gov. National Library of Medicine (US). Testing the Addition of the Drug Atezolizumab to the Usual Radiation Treatment for Patients With Early Nonsmall Cell Lung Cancer. Identifier: NCT04214262. Available online: https://www.clinicaltrials.gov/ct2/show/ NCT04214262. [accessed 4.19.2020]. 